\title{
Prebiotics and Probiotics: A Panacea for Chronic Health Problems?
}

\section{Thaddeus Ezeji*}

Biotechnology and Fermentation Group, The Ohio State University, USA

Owing to distinctive evidence depicting the health-promoting properties of functional foods, their consumption has garnered significant global interest. Consequently, the functional food market is growing rapidly and dairy-based functional foods (yogurt, fermented dairy drinks, cheese and ice-cream) are at the forefront of this growth. The concept of probiotics was introduced by a Russian Nobel laureate, Professor Elie Metchnikoff, who famously promoted the idea that Bulgarian peasants who were known to live healthy and long lives regularly consumed fermented milk (yogurt). He documented that the bacterial constituents of the yogurt were responsible for the good health enjoyed by these consumers. Majority of the commonly known probiotics belong to four genera namely Lactobacillus (L. acidophilus, L. casei, L. delbrueckii, L. plantarum, L. rhamnosus), Bifidobacterium (B. bifidum, B. Breve, B. Infantis, B. Longum, B. lactis), Streptococcus (S. thermophilus, S. salivarius, S. diacetylactis, and S. intermedius), and Bacillus (B. cereus, B. clausii, B. pumilus, B. coagulans). Given that some strains of these species do not have any benefit and potentially pathogenic at worst, certain criteria such as safety, established or confirmed efficacious dose, viability of the culture at the time of consumption and established health benefits must be met before a microorganism can be considered to be 'probiotic'.

While prebiotics have been recognized in the early 1950s when György described "bifidus factor", an ingredient that selectively promoted the growth of bifidobacteria (formerly called Lactobacillus bifidus) [1,2], the term "prebiotic" was coined in 1995 by Gibson and Roberfroid [3]. Prebiotics are non-digestible dietary supplements, which beneficially adjust intestinal microflora by stimulating the growth and activity of beneficial microorganisms (probiotics) in the host organism. It is important to note that humans do not possess the transport systems and accompanying enzymes necessary for the metabolism of prebiotics. Consequently, prebiotics are completely available to the probiotics that reside in the gastrointestinal tract. Some of the commonly known prebiotics are lactose, inulin (with low degree of polymerization), fructo-oligosacccharides, arabinose, xylose and xylo-oligosaccharides.

By combining probiotics and prebiotics, a product often referred to as synbiotics or eubiotics, is obtained. These products deliver live exogenous beneficial microbes in the gastrointestinal flora, which subsequently colonize the gastrointestinal tract (stomach and large intestines) to enhance their survival. Notably, many of bacterial metabolites are absorbed into the blood stream of the host. Passing through the chyme/blood barrier, they enter the systemic body space where they interact with various physiological processes in vital organs and peripheral tissues of the host, resulting in enhanced digestion, bone density, immunity and overall health [4].

With the advances in the field of probiotics and prebiotics along with heightened awareness of their related health benefits, one wonders if this area of endeavor could be expanded and exploited further. Given the global high rate of obesity that cuts across all age groups and the related chronic disease conditions, development of functional foods that can mitigate obesity problems is crucial. Since xylose, a prebiotic that is non-digestible by humans, is sweeter than glucose, it has become imperative for functional food companies and food formulators to find sundry means to substitute significant portions of glucose in high calorie foods with xylose. Currently, the limited use of xylose only as a sweetener in special foods and as a remedy for certain health problems is inadequate. Efficient use of xylose and other natural nonmetabolizable sugars along with compatible probiotics in food may not be the panacea for the global obesity problems; however, it will almost certainly have a positive impact on disease resistance, mitigation of obesity problems, and other chronic health problems.

\section{References}

1. Gyorgy P, Norris RF, Rose CS (1954) Bifidus factor. I. A variant of Lactobacillus bifidus requiring a special growth factor. Arch Biochem Biophys 48: 193-201.

2. Gauhe A, Gyorgy P, Hoover JR, Kuhn R, Rose CS, et al. (1954) Bifidus factor IV. Preparations obtained from human milk. Arch Biochem Biophys 48: 214224.

3. Gibson GR, Roberfroid MB (1995) Dietary modulation of the human colonic microbiota: introducing the concept of prebiotics. J Nutr 125: 1401-1412.

4. Lenoir-Wijnkoop I, Sanders ME, Cabana MD, Caglar E, Corthier G, et al. (2007) Probiotic and prebiotic influence beyond the intestinal tract. Nutr Rev 65: 469489 .
*Corresponding author: Thaddeus Ezeji, Biotechnology and Fermentation Group, The Ohio State University, USA, Tel: 1-330-263-3796; Fax: 1-330-2633949; E-mail: ezeji.1@osu.edu

Received July 28, 2012; Accepted July 28, 2012; Published July 29, 2012

Citation: Ezeji T (2012) Prebiotics and Probiotics: A Panacea for Chronic Health Problems? J Food Process Technol 3:e107. doi:10.4172/2157-7110.1000e107

Copyright: (c) 2012 Ezeji T. This is an open-access article distributed under the terms of the Creative Commons Attribution License, which permits unrestricted use, distribution, and reproduction in any medium, provided the original author and source are credited. 